\title{
Synthesis and Antimicrobial Activity of Novel Phosphorus Heterocycles with Exocyclic P-C Link
}

\author{
Peddaiahgari Vasu Govardhana Reddy, Yarragudi Bathal Reddy Kiran, Cirandur Suresh Reddy, ${ }^{*}$ \\ and Chichili Devendranath RedDY
}

Department of Chemistry, Sri Venkateswara University College of Engineering; Tirupati-517 502, India.

Received August 22, 2003; accepted December 22, 2003

\begin{abstract}
Several new class of phosphorus heterocyclic compounds containing exocyclic P-C link such as 6-(2'chloroethyl)/(allyl)/(benzyl)-1,2,4,8,10,11-hexachloro-12H-dibenzo[d,g][1,3,2]dioxaphosphocin 6-oxides (5-7), 2(2"-chloroethyl)/(allyl)-6-(1,1-dimethylethyl)-3-cyclohexyl-3,4-dihdro-2H-1,3,2-benzoxazaphosphorin 2-oxides (9, 10), 2-(2"-chloroethyl-2,3-dihydro-3-(4'-bromophenyl)-1H-naphth[1,2-e][1,3,2]-oxazaphosphorin 2-oxide (12), 2-(2"-chloroethyl)/(allyl)-2,3-dihydro-5-benzoyl-1H-1,3,2-benzodiazaphosphole 2-oxides (14, 15), 4-phenyl-2(2"-chloroethyl)-1H-1,3,3a,5,6-pentaza-2-phosphapentalene 2-oxide (17) and 4-benzyl-2-(2"-chloroethyl)-1H1,3,3a,5,6-pentaza-2-phospha-pentalene 2-oxide (19) were synthesized by reacting equimolar quantities of corresponding diol (4)/diamines (13, 16, 18), 2-cyclohexylaminomethyl-4-t-butylphenol (8) and 1-(4'-bromoanilinomethyl)-2-naphthol (11), with respective phosponyl dichlorides $(1-3)$ in dry toluene/toluene-tetrahydrofuran/pyridine in the presence of triethylamine at various temperatures. Their structures were established by IR, ${ }^{1} \mathrm{H}-,{ }^{13} \mathrm{C}$ - and ${ }^{31} \mathrm{P}-\mathrm{NMR}$ spectral data. The mass spectral data were given for compounds 9,12 and 15 . The title compounds were screened for antibacterial activity against Staphylococcus aureus and Escherichia coli and antifungal activity on Aspergillus niger and Helminthosporium oryzae. Most of the compounds possess significant activity.
\end{abstract}

Key words phosphonyl dichloride; hexachlorophene; 1-(4-bromoanilinomethyl)-2-naphthol; 2-cyclohexylaminomethyl-4-tbutylphenol; 3,4-diaminobenzophenone; 5-phenyl-1,2-diamino-1,3,4-triazole

In the realm of organophosphorus chemistry, phosphonates are interesting complements, in terms of biological activity. ${ }^{1)}$ They play an important and useful role as inhibitors of gene expression in mammalian cells ${ }^{2)}$ and as antibiotics. ${ }^{3)}$ They have been also developed to be used as insecticides, ${ }^{3)}$ herbicides, ${ }^{3,4)}$ fungicides ${ }^{3,5)}$ and plant growth regulators. $\left.{ }^{4}\right) \mathrm{Re}-$ cently, they gained importance in the treatment of bone disorders. ${ }^{6}$

Denmark and Hua synthesized exocyclic P-C link compounds as cis and trans 3-substituted-2-benzyl-6-methyl1,3,2-oxazaphosphorinane 2-oxides ${ }^{7)}$ and 2-allyl-1,3,2-oxazaphospholidine 2-oxides. ${ }^{8)}$ In our search for eco/bio-friendly pesticides, we have reported synthesis of several exocyclic $\mathrm{P}-\mathrm{O}$ and $\mathrm{P}-\mathrm{NH}$ link dioxaphosphocins, ${ }^{9}$ ) oxazaphosporins ${ }^{10,11)}$ and benzodiazaphospholes. ${ }^{12}$ In continuation of our endeavour to develop potential pesticides, a new group of exocyclic $\mathrm{P}-\mathrm{C}$ link compounds were synthesized.

1,2-Dichloroethane, allyl chloride and benzyl chloride reacted readily with phosphorus trichloride in the presence of aluminium chloride to give complexes, which on treatment with water gave good yields of 2-chloroethyl phosphonyl dichloride (1), allyl phosphonyl dichloride (2) and benzyl phosphonyl dichloride (3) (Chart 1), ${ }^{13}$ respectively. But, vinylchloride did not react in a similar way to yield vinyl phosphonyl dichloride due to its inability to form the com-

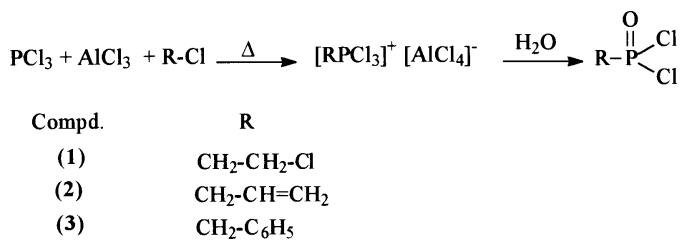

Chart 1 plex with aluminium chloride because of extended $\pi$-electron delocalisation.

Synthesis of $5,6,7,9,10,12,14,15,17$ and 19 was achieved by the cyclocondensation of equimolar quantities of hexachlorophene (4), 2-cyclohexylaminomethyl-4-t-butylphenol $^{14)}$ (8), 1-(4-bromoanilinomethyl)-2-naphthol ${ }^{15)}$ (11), 3,4-diaminiobenzophenone (13), 5-phenyl-1,2-diamino-1,3,4triazole $^{16)}$ (16) and 3-benzyl-4,5-diamino-1,2,4-triazole ${ }^{17)}$ (18) with 2-chloroethyl phosphonyl dichloride (1), allyl phosphonyl dichloride (2) and benzyl phosphonyl dichloride (3) in dry toluene/toluene-tetrahydrofuran/pyridine at various temperatures (Chart 2). Purifications of compounds were achieved by filtering off the triethylamine hydrochloride, evaporation of the filtrate, washing of the residue with water and recrystallization of the solid products employing suitable solvents (ethyl acetate-hexane/aqueous 2-propanol). Their structures were established by IR, ${ }^{1} \mathrm{H}-,{ }^{13} \mathrm{C}-,{ }^{31} \mathrm{P}-\mathrm{NMR}$ and mass spectra.

The bridged methylene protons $\left(12-\mathrm{CH}_{2}\right)$ of $\mathbf{5}, \mathbf{6}$ and 7 showed two distinct doublets in the regions $\delta 4.36-4.42$ and $\delta 4.81-4.86$ due to geminal coupling. The expected longrange coupling $\left[J_{(\mathrm{H}, \mathrm{P})}\right]$ between phosphorus and one of the bridged methylene protons $\left(12-\mathrm{CH}_{2}\right)$ was not observed. ${ }^{18,19)}$ The bridged methylene protons $\left(12-\mathrm{CH}_{2}\right)$ in certain dibenzodioxaphosphocins, which are largely in the boat-chair conformation, exhibit only geminal coupling $\left[J_{(\mathrm{H}, \mathrm{H}}\right]$ and do not show long-range coupling with phosphorus $\left[J_{(\mathrm{H}, \mathrm{P})}\right]{ }^{20)}$ The $J_{(\mathrm{H}, \mathrm{P})}$ coupling would not be expected for molecules in a boat-chair conformation with the phosphoryl oxygen in a pseudo equatorial position, based upon the through space mechanism for transmission of coupling information, involving the lone pair of electrons of the endocyclic oxygen atoms. ${ }^{21)}$ On the same basis, it may be construed that the compounds 5, 6 and 7 may have the boat-chair (BC) confor- 


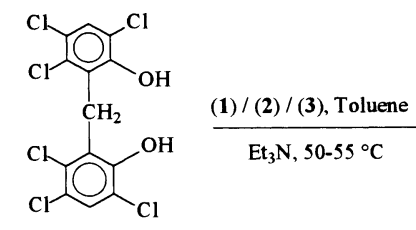

(4)

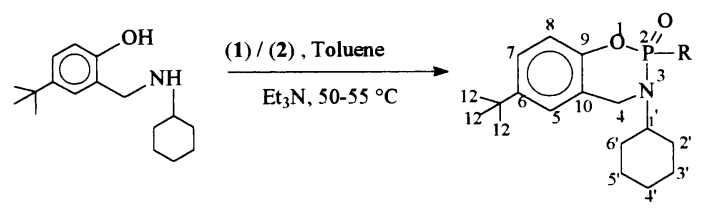

(8)

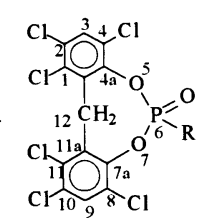

(5-7)

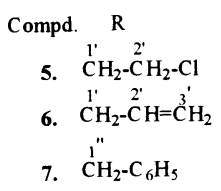
9. $\mathrm{CH}_{2}-\mathrm{CH}_{2}-\mathrm{Cl}$ 10. $\stackrel{1}{\mathrm{C}} \mathrm{H}_{2}-\stackrel{2}{\mathrm{C}} \mathrm{H}=\stackrel{3 "}{\mathrm{C}} \mathrm{H}_{2}$

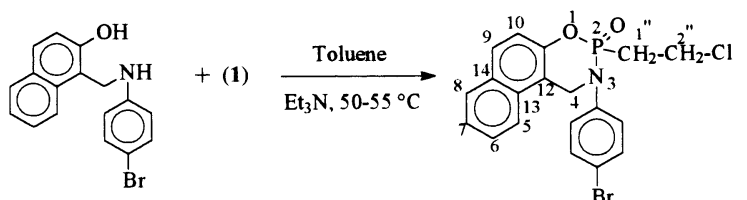

(11)

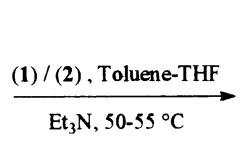

(13)<smiles>[R][Y7]1([H])Nc2ccc(C(=O)c3ccccc3)cc2N1P</smiles>

(14-15)
$\mathrm{R}$

14. $\stackrel{1 "}{\mathrm{C}} \stackrel{2}{\mathrm{H}_{2}}-\stackrel{2 "}{\mathrm{C}} \mathrm{H}_{2}-\mathrm{Cl}$

15. $\stackrel{1}{\mathrm{C}} \mathrm{H}_{2}-\stackrel{2}{\mathrm{C}} \mathrm{H}=\stackrel{3}{\mathrm{C}} \mathrm{CH}_{2}$

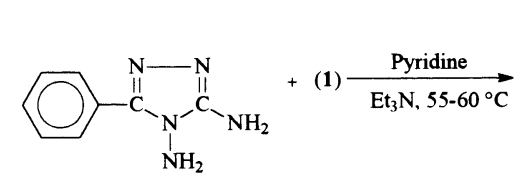

(16)<smiles>CN1N(C)P(O)(P)(CCCl)Nc2nnc(-c3ccccc3)n21</smiles>

(17)

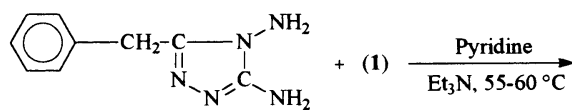

(18)

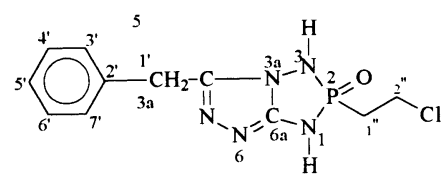

(19)

Chart 2

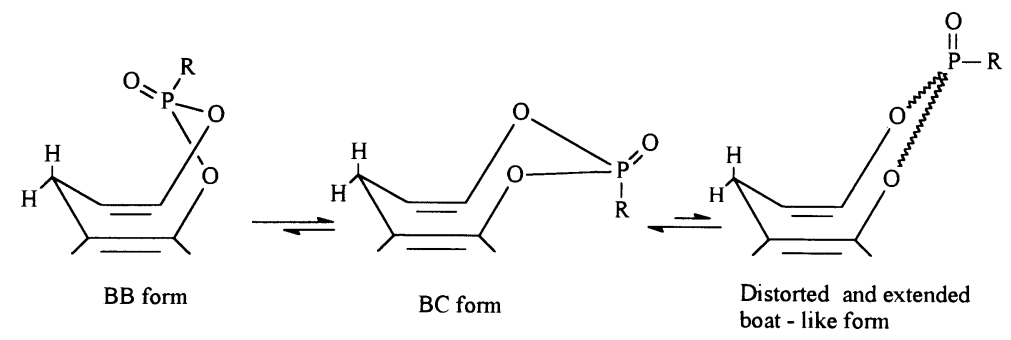

Fig. 1

mation, which is in rapid conformational equilibrium with either a boat-boat (BB) or a distorted and extended boat-like form $^{20)}$ (Fig. 1).

In compounds 9 and $\mathbf{1 0}$ the methylene $(\mathrm{C}-4)$ protons of the oxazaphosphorin ring moiety resonated as multiplets ${ }^{22)}$ at $\delta$ $4.00-4.06$ and $\delta 3.97-4.01$ as a result of induced coupling with phosphorus. In compound 12, the C-4 methylene protons gave two multiples at $\delta 4.96-5.03$ and $\delta 5.37-5.47$ respectively, suggesting that they are magnetically nonequivalent due to their axial and equatorial orientations in the

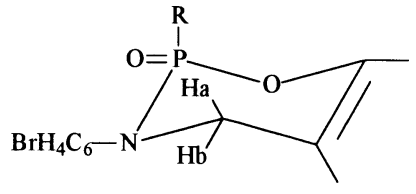

Fig. 2

six-membered chair conformation of the benzoxazaphosphorin ring ${ }^{10)}$ (Fig. 2).

Antimicrobial Activity All the compounds 5, 6, 7, 9, 
Table 1. Antimicrobial Activity of 5, 6, 7, 9, 10, 12, 14, 15, 17 and 19

\begin{tabular}{|c|c|c|c|c|c|c|c|c|}
\hline \multirow{4}{*}{ Compd. } & \multicolumn{8}{|c|}{ Zone of inhibition (mm) } \\
\hline & \multicolumn{4}{|c|}{ Bacteria } & \multicolumn{4}{|c|}{ Fungi } \\
\hline & \multicolumn{2}{|c|}{ Staphylococcus aureus } & \multicolumn{2}{|c|}{ Escherichia coli } & \multicolumn{2}{|c|}{ Aspergillus niger } & \multicolumn{2}{|c|}{ Helminthosporium oryzae } \\
\hline & $\begin{array}{c}250 \\
(\mu \mathrm{g} / \mathrm{disc})\end{array}$ & $\begin{array}{c}500 \\
(\mu \mathrm{g} / \mathrm{disc})\end{array}$ & $\begin{array}{c}250 \\
(\mu \mathrm{g} / \mathrm{disc})\end{array}$ & $\begin{array}{c}500 \\
(\mu \mathrm{g} / \mathrm{disc})\end{array}$ & $\begin{array}{c}250 \\
(\mu \mathrm{g} / \mathrm{disc})\end{array}$ & $\begin{array}{c}500 \\
(\mu \mathrm{g} / \mathrm{disc})\end{array}$ & $\begin{array}{c}250 \\
(\mu \mathrm{g} / \text { disc })\end{array}$ & $\begin{array}{c}500 \\
(\mu \mathrm{g} / \mathrm{disc})\end{array}$ \\
\hline 5 & 14 & 18 & 16 & 19 & 18 & 26 & 20 & 26 \\
\hline 6 & 12 & 15 & 12 & 17 & 15 & 24 & 15 & 20 \\
\hline 7 & 13 & 19 & 10 & 15 & 16 & 22 & 14 & 18 \\
\hline 9 & 5 & 8 & 4 & 6 & 10 & 16 & 10 & 15 \\
\hline 10 & $ـ^{a)}$ & - & - & - & 12 & 18 & 9 & 13 \\
\hline 12 & 10 & 16 & 8 & 15 & 14 & 20 & 12 & 18 \\
\hline 14 & 6 & 9 & 4 & 8 & 10 & 15 & 8 & 12 \\
\hline 15 & - & - & - & - & 9 & 14 & 6 & 10 \\
\hline 17 & 12 & 16 & 8 & 15 & 12 & 16 & 10 & 17 \\
\hline 19 & 10 & 14 & 12 & 16 & 8 & 12 & 12 & 18 \\
\hline Penicillin & 22 & & 21 & & & & & \\
\hline Streptomycin & 27 & & 25 & & & & & \\
\hline Griseofulvin & & & & & 28 & & 28 & \\
\hline
\end{tabular}

a) — indicates no activity.

10, 12, 14, 15, 17 and 19 (Table 1) were screened for their antibacterial activity against Staphylococcus aureus and Escherichia coli $\left(10^{6} \mathrm{cell} / \mathrm{ml}\right)$ by the disc-diffusion method ${ }^{23)}$ in nutrient agar medium. Various concentrations of synthesized compounds $(250,500 \mu \mathrm{g} / \mathrm{disc})$ dissolved in dimethylformamide (DMF) were added to each filter disc and DMF was used as control. Plates were incubated at $37^{\circ} \mathrm{C}$ and examined for zone of inhibition around each disc after $24 \mathrm{~h}$. The results were compared with standard antibiotics like penicillin and streptomycin $(250 \mu \mathrm{g} /$ disc $)$. Their antifungal activity ${ }^{24)}$ was evaluated against Aspergillus niger and Helminthosporium oryzae at concentrations of 250 and $500 \mu \mathrm{g} / \mathrm{disc}$. Griseofulvin was used as reference compound. The fungal cultures were grown on potato dextrose broth at $25^{\circ} \mathrm{C}$ for $3 \mathrm{~d}$ and finally spore suspension was adjusted to $10^{5} \mathrm{spores} / \mathrm{ml}$. Most of the compounds showed significant activity against both bacteria and fungi.

In summary, we have reported an effective and simple route for the synthesis of novel compounds with exocyclic $\mathrm{P}-\mathrm{C}$ linkage and proved that dibenzodioxaphosphocin ring system exist preferentially in boat-chair conformation.

\section{Experimental}

Melting points determined on a Mel-Temp apparatus were uncorrected. IR spectra were recorded in $\mathrm{KBr}$ pellets on a Perkin-Elmer 1430 unit. The ${ }^{1} \mathrm{H}-$, ${ }^{13} \mathrm{C}$ - and ${ }^{31} \mathrm{P}$-NMR spectra were taken on AMX $400 \mathrm{MHz}$ spectrometer operating at $400 \mathrm{MHz}$ for ${ }^{1} \mathrm{H}, 100 \mathrm{MHz}$ for ${ }^{13} \mathrm{C}$ and $161.9 \mathrm{MHz}$ for ${ }^{31} \mathrm{P}$. Compounds were referenced from TMS $\left({ }^{1} \mathrm{H}\right.$ and ${ }^{13} \mathrm{C}, \delta$ or ppm). All ${ }^{31} \mathrm{P}-\mathrm{NMR}$ data were taken on similar solutions and referenced to $85 \% \mathrm{H}_{3} \mathrm{PO}_{4}\left({ }^{31} \mathrm{P}, \delta\right.$ or $\mathrm{ppm})$. Mass spectral data were recorded for 12 on GC-MS at $70 \mathrm{eV}$, with a direct inlet system. General procedures for the preparations of $\mathbf{5}, \mathbf{6}, \mathbf{7}, \mathbf{9}, \mathbf{1 0}$, $12,14,15,17$ and 19 are illustrated with the synthesis of 5 .

Hexachlorophene (4), 3,4-diaminobenzophenone (13) were procured from Aldrich Chemical Company, Inc, U.S.A. and were used without further purification. 8, 11, 16 and $\mathbf{1 8}$ compounds were prepared according to the reported procedures.

Synthesis of 6-(2'-Chloroethyl)-1,2,4,8,10,11-hexachloro-12H-dibenzo[d,g][1,3,2]dioxaphosphocin 6-Oxide (5) 2-Chloroethyl phosphonyl dichloride $(1,0.36 \mathrm{~g}, 2 \mathrm{mmol})$ in dry toluene $(20 \mathrm{ml})$ was added to the stirred solution of hexachlorophene $(4,0.812 \mathrm{~g}, 2 \mathrm{mmol})$ and triethylamine $(0.404 \mathrm{~g}$, $4 \mathrm{mmol})$ in dry toluene $(40 \mathrm{ml})$ over a period of twenty minutes at $0^{\circ} \mathrm{C}$. The reaction mixture was slowly heated to $50-55^{\circ} \mathrm{C}$ and stirring was continued for $5 \mathrm{~h}$. The progress of the reaction was monitored by thin layer chromatography (TLC) in the 1:4 mixture of ethyl acetate and hexane as mobile solvent. The reaction mixture was filtered to separate triethylamine hydrochloride and the solvent in the filtrate was removed off under reduced pressure. The crude cyclized product after washing with water and drying was recrystallized from ethyl acetate-hexane to obtain pure 5. Yield $0.67 \mathrm{~g}(65 \%)$, mp $150-152^{\circ} \mathrm{C}$. Anal. Calcd for $\mathrm{C}_{15} \mathrm{H}_{8} \mathrm{PO}_{3} \mathrm{Cl}_{7} ; \mathrm{C}, 34.96 ; \mathrm{H}, 1.56$. Found: $\mathrm{C}$, 34.82; H, 1.68. IR (KBr) cm ${ }^{-1}: 1280(\mathrm{P}=\mathrm{O}), 736(\mathrm{P}-\mathrm{C}) .{ }^{31} \mathrm{P}-\mathrm{NMR}\left(\mathrm{CDCl}_{3}\right)$ $\delta$ : 22.0. ${ }^{1} \mathrm{H}-\mathrm{NMR}\left(\mathrm{CDCl}_{3}\right) \delta: 7.55(\mathrm{~s}, 2 \mathrm{H}, \mathrm{C}-3,9-\mathrm{H}, \mathrm{Ar}-\mathrm{H}),[4.86(\mathrm{~d}$, $J=15.9 \mathrm{~Hz}, 1 \mathrm{H}, \mathrm{Ha}), 4.36(\mathrm{~d}, J=15.9 \mathrm{~Hz}, 1 \mathrm{H}, \mathrm{Hb})$ : bridged $\left.\mathrm{CH}_{2}\right], 3.98$ $4.05\left(\mathrm{~m}, 2 \mathrm{H}, \mathrm{CH}_{2} \mathrm{Cl}\right), 2.82-2.91\left(\mathrm{~m}, 2 \mathrm{H}, \mathrm{P}-\mathrm{CH}_{2}\right) .{ }^{13} \mathrm{C}-\mathrm{NMR}\left(\mathrm{CDCl}_{3}\right) \delta$ : 133.2 (s, 2C, C-1, 11), 131.6 (s, 2C, C-2, 10), 130.4 (d, J=3.2 Hz, 2C, C-3, 9), 125.0 (d, $J=6 \mathrm{~Hz}, 2 \mathrm{C}, \mathrm{C}-4,8), 146.1$ (d, $J=10.5 \mathrm{~Hz}, 2 \mathrm{C}, \mathrm{C}-4 \mathrm{a}, 7 \mathrm{a}), 129.4$ (s, 2C, C-11a, 12a), 29.7 (s, 1C, C-12, bridged $\left.\mathrm{CH}_{2}\right), 32.7$ (d, $\left.{ }^{1} J_{\mathrm{PC}}=132.8 \mathrm{~Hz}, 1 \mathrm{C}, \mathrm{C}-1^{\prime}, \mathrm{P}-\mathrm{CH}_{2}\right), 36.3\left(\mathrm{~s}, 1 \mathrm{C}, \mathrm{C}-2^{\prime}, \mathrm{CH}_{2} \mathrm{Cl}\right)$.

This procedure was used for the preparation of $6,7,9,10,12,14,15,17$ and 19 compounds. The physical and spectroscopic data ${ }^{25)}$ of all the compounds are given below.

6-(Allyl)-1,2,4,8,10,11-hexachloro-12H-dibenzo[ $[d, g][1,3,2]$ dioxaphosphocin 6-Oxide (6) Yield: $0.55 \mathrm{~g}(56 \%)$; mp $106-108^{\circ} \mathrm{C}$. Anal. Calcd for $\mathrm{C}_{16} \mathrm{H}_{9} \mathrm{PO}_{3} \mathrm{Cl}_{6}: \mathrm{C}, 38.98 ; \mathrm{H}, 1.84$. Found: $\mathrm{C}, 38.79 ; \mathrm{H}, 1.56$. IR $(\mathrm{KBr}) \mathrm{cm}^{-1}$ : $1234(\mathrm{P}=\mathrm{O}), 737(\mathrm{P}-\mathrm{C}) .{ }^{31} \mathrm{P}-\mathrm{NMR}\left(\mathrm{DMSO}-d_{6}\right) \delta: 24.51 .{ }^{1} \mathrm{H}-\mathrm{NMR}$ (DMSO$\left.d_{6}\right) \delta: 7.75(\mathrm{~s}, 2 \mathrm{H}, \mathrm{C}-3,9-\mathrm{H}, \mathrm{Ar}-\mathrm{H}),[4.81(\mathrm{~d}, J=16.2 \mathrm{~Hz}, 1 \mathrm{H}, \mathrm{Ha}), 4.39(\mathrm{~d}$, $J=16.2 \mathrm{~Hz}, 1 \mathrm{H}, \mathrm{Hb})$; bridged $\left.\mathrm{CH}_{2}\right], 2.58-2.87\left(\mathrm{~m}, 2 \mathrm{H}, \mathrm{P}-\mathrm{CH}_{2}\right), 5.73-5.87$ $(\mathrm{m}, 1 \mathrm{H}, \mathrm{CH}), 5.12\left(\mathrm{~d}, J_{\text {trans }}=16.7 \mathrm{~Hz}, \mathrm{CH}_{2}\right), 5.04\left(\mathrm{~d}, J_{\text {cis }}=9.8 \mathrm{~Hz}, \mathrm{CH}_{2}\right), 7.59$ and $7.60\left(2 \mathrm{~d}, J_{\text {gem }}=2.7,2.4 \mathrm{~Hz}, \mathrm{CH}_{2}\right) .{ }^{13} \mathrm{C}-\mathrm{NMR}$ (DMSO- $\left.d_{6}\right) \delta: 134.3(\mathrm{C}-1$, 11), 130.6 (C-2, 10), 129.4 (C-3, 9), 126.2 (C-4, 8), 151.5 (C-4a, 7a), 128.7 $(\mathrm{C}-11 \mathrm{a}, 12 \mathrm{a}), 33.9\left(\mathrm{C}-12\right.$, bridged $\left.\mathrm{CH}_{2}\right), 31.3\left(\mathrm{~d},{ }^{1} J_{\mathrm{PC}}=160.0 \mathrm{~Hz}, \mathrm{C}-1^{\prime}\right.$, $\left.\mathrm{P}-\mathrm{CH}_{2}\right), 134.3\left(\mathrm{C}-2^{\prime}\right), 117.5\left(\mathrm{~d},{ }^{3} J_{\mathrm{PC}}=14 \mathrm{~Hz}, \mathrm{C}-3^{\prime}\right)$.

6-(Benzyl)-1,2,4,8,10,11-hexachloro-12H-dibenzo $[d, g][1,3,2]$ dioxaphosphocin 6-Oxide (7) Yield: $0.55 \mathrm{~g}(51 \%)$; mp $152-154^{\circ} \mathrm{C}$. Anal. Calcd for $\mathrm{C}_{20} \mathrm{H}_{11} \mathrm{PO}_{3} \mathrm{Cl}_{6}$ : C, 44.23; H, 2.04. Found: $\mathrm{C}, 44.45 ; \mathrm{H}, 2.19$. IR $(\mathrm{KBr}) \mathrm{cm}^{-1}$ : $1287(\mathrm{P}=\mathrm{O}), 730(\mathrm{P}-\mathrm{C}) .{ }^{31} \mathrm{P}-\mathrm{NMR}\left(\mathrm{DMSO}-d_{6}\right) \delta: 23.94 .{ }^{1} \mathrm{H}-\mathrm{NMR}$ (DMSO$\left.d_{6}\right) \delta: 7.60(\mathrm{~s}, 2 \mathrm{H}, \mathrm{C}-3,9-\mathrm{H}),[4.84(\mathrm{~d}, J=16.0 \mathrm{~Hz}, 1 \mathrm{H}, \mathrm{Ha}), 4.42(\mathrm{~d}$, $J=16.0 \mathrm{~Hz}, 1 \mathrm{H}, \mathrm{Hb})$; bridged $\left.\mathrm{CH}_{2}\right], 7.21-7.74(\mathrm{~m}, 5 \mathrm{H}, \mathrm{Ar}-\mathrm{H}), 3.21$ (d, $\left.J_{\mathrm{PH}}=20.2 \mathrm{~Hz}, \mathrm{P}-\mathrm{CH}_{2}\right) .{ }^{13} \mathrm{C}-\mathrm{NMR}\left(\mathrm{DMSO}-d_{6}\right) \delta: 134.3(\mathrm{C}-1,11), 131.0(\mathrm{C}-$ 2, 10), 129.7 (C-3, 9), 125.8 (C-4, 8), 151.6 (C-4a, 7a), 128.7 (C-11a, 12a), $29.7\left(\mathrm{C}-12\right.$, bridged $\left.\mathrm{CH}_{2}\right), 31.3\left(\mathrm{~d}, J_{\mathrm{PC}}=162.9 \mathrm{~Hz}, \mathrm{C}-1^{\prime}, \mathrm{P}-\mathrm{CH}_{2}\right), 134.3(\mathrm{C}-$ $\left.2^{\prime}\right), 128.6\left(\mathrm{C}-3^{\prime}, 7^{\prime}\right), 127.7\left(\mathrm{C}-4^{\prime}, 6^{\prime}\right), 126.3\left(\mathrm{C}-5^{\prime}\right)$

2-(2"-Chloroethyl)-6-(1,1-dimethylethyl)-3-cyclohexyl-3,4-dihydro-2H1,3,2-benzoxazaphosphorin 2-Oxide (9) Yield: 0.46g (62\%); mp 203$205^{\circ}$ C. Anal. Calcd for $\mathrm{C}_{19} \mathrm{H}_{29} \mathrm{NPO}_{2} \mathrm{Cl}: \mathrm{C}, 61.68 ; \mathrm{H}, 7.90 ; \mathrm{N}, 3.78$. Found: $\mathrm{C}, 61.82 ; \mathrm{H}, 7.73 ; \mathrm{N}, 3.86$. IR $(\mathrm{KBr}) \mathrm{cm}^{-1}: 1262(\mathrm{P}=\mathrm{O}), 742(\mathrm{P}-\mathrm{C}) .{ }^{31} \mathrm{P}-$ NMR $\left(\mathrm{CDCl}_{3}\right) \delta: 16.00 .{ }^{1} \mathrm{H}-\mathrm{NMR}\left(\mathrm{CDCl}_{3}\right) \delta: 6.85-7.48(\mathrm{~m}, 3 \mathrm{H}, \mathrm{Ar}-\mathrm{H})$, $4.00-4.06\left(\mathrm{~m}, 2 \mathrm{H}, 4-\mathrm{CH}_{2}\right), 1.26(\mathrm{~s}, 9 \mathrm{H}, t$-butyl-H), $1.45-1.78(\mathrm{~m}, 11 \mathrm{H}$, Cyclohexyl-H), 3.79-3.85 (m, 2H, CH $\mathrm{Cl}_{2} \mathrm{Cl}, 2.51-2.68\left(\mathrm{~m}, 2 \mathrm{H}, \mathrm{P}-\mathrm{CH}_{2}\right)$ ${ }^{13} \mathrm{C}-\mathrm{NMR}\left(\mathrm{CDCl}_{3}\right) \delta: 43.6(\mathrm{C}-4), 123.8(\mathrm{C}-5), 147.1(\mathrm{C}-6), 126.3(\mathrm{C}-7)$, 
$118.2(\mathrm{~d}, J=7.1 \mathrm{~Hz}), 147.6(\mathrm{~d}, J=7.4 \mathrm{~Hz}, \mathrm{C}-9), 124.5(\mathrm{~s}, 1 \mathrm{C}, \mathrm{C}-10), 34.7(\mathrm{C}-$ 11), 31.9 (C-12), $55.6\left(\mathrm{C}-1^{\prime}\right), 32.1\left(\mathrm{C}-2^{\prime}, 6^{\prime}\right), 25.8$ (C-3', 5'), $25.2\left(\mathrm{C}-4^{\prime}\right)$, $31.4\left(\mathrm{~d},{ }^{1} J_{\mathrm{PC}}=145.1 \mathrm{~Hz}, \mathrm{C}-1^{\prime \prime}, \mathrm{P}-\mathrm{CH}_{2}\right), 36.3\left(\mathrm{C}-2^{\prime \prime}, \mathrm{CH}_{2} \mathrm{Cl}\right)$. EI-MS $m / z(\%)$ $347\left(13, \mathrm{M}^{+}\right.$), 291 (30), 262 (65), 245 (37), 219 (40), 164 (56), 147 (38), 91 (31), 56 (100).

2-(Allyl)-6-(1,1-dimethylethyl)-3-cyclohexyl-3,4-dihydro-2H-1,3,2-benzoxazaphosphorin 2-Oxide (10) Yield: $0.41 \mathrm{~g}(58 \%)$; $\mathrm{mp} 186-188^{\circ} \mathrm{C}$ Anal. Calcd for $\mathrm{C}_{20} \mathrm{H}_{30} \mathrm{NPO}_{2}$ : C, 68.26; H, 8.95; N, 3.98. Found: C, 68.49; $\mathrm{H}, 8.26 ; \mathrm{N}$, 3.98. IR $(\mathrm{KBr}) \mathrm{cm}^{-1}: 1224(\mathrm{P}=\mathrm{O}), 736(\mathrm{P}-\mathrm{C}) .{ }^{31} \mathrm{P}-\mathrm{NMR}$ $\left(\mathrm{DMSO}_{-} d_{6}\right) \delta: 18.48 .{ }^{1} \mathrm{H}-\mathrm{NMR}\left(\mathrm{DMSO}-d_{6}\right) \delta: 7.02-7.42(\mathrm{~m}, 5 \mathrm{H}, \mathrm{Ar}-\mathrm{H}+$ olefinic-H), $1.13(\mathrm{~s}, 9 \mathrm{H}, t$-butyl-H), 1.60-2.10 (m, 11H, Cyclohexyl-H), $3.97-4.01\left(\mathrm{~m}, 2 \mathrm{H}, 4-\mathrm{CH}_{2}\right), 3.62-3.90\left(\mathrm{~m}, 2 \mathrm{H}, \mathrm{P}-\mathrm{CH}_{2}\right), 5.04-5.93(\mathrm{~m}$, $1 \mathrm{H}, \mathrm{CH}), 5.09\left(\mathrm{~d}, J_{\text {trans }}=17.6 \mathrm{~Hz}, 2 \mathrm{H}, \mathrm{CH}_{2}\right), 5.92\left(\mathrm{~d}, J_{c i s}=9.5 \mathrm{~Hz}, 2 \mathrm{H}, \mathrm{CH}_{2}\right)$ ${ }^{13} \mathrm{C}-\mathrm{NMR}$ (DMSO- $d_{6}$ ) $\delta$ : 42.9 (C-4), 124.4 (C-5), 149.3 (C-6), 126.9 (C-7), 117.7 (C-8), 145.7 (C-9), 122.7 (C-10), 34.4 (C-11), 33.1 (C-12), 55.4 (C$\left.1^{\prime}\right), 33.9\left(\mathrm{C}-2^{\prime}, 6^{\prime}\right), 24.8\left(\mathrm{C}-3^{\prime}, 5^{\prime}\right), 24.0\left(\mathrm{C}-4^{\prime}\right), 31.3\left(\mathrm{~d},{ }^{1} J_{\mathrm{PC}}=156.2 \mathrm{~Hz}, \mathrm{C}-\right.$ $\left.1^{\prime \prime}, \mathrm{P}-\mathrm{CH}_{2}\right), 132.4\left(\mathrm{C}-2^{\prime \prime}\right), 116.7\left(\mathrm{~d},{ }^{3} J_{\mathrm{PC}}=13.3 \mathrm{~Hz}, \mathrm{C}-3^{\prime \prime}\right)$.

2-(2"-Chloroethyl)-2,3-dihydro-3-(4'-bromophenyl)-1H-naphth[1,2e][1,3,2]oxazaphosphorin 2-Oxide (12) Yield: $0.59 \mathrm{~g}$ (68\%); mp 235$237^{\circ} \mathrm{C}$. Anal. Calcd for $\mathrm{C}_{19} \mathrm{H}_{16} \mathrm{NPO}_{2} \mathrm{ClBr}$ : C, 52.26; H, 3.69; N, 3.20. Found: C, 52.08; H, 3.54; N, 3.09. IR (KBr) cm $\mathrm{cm}^{-1}: 1217(\mathrm{P}=\mathrm{O}), 744(\mathrm{P}-\mathrm{C})$. ${ }^{31} \mathrm{P}-\mathrm{NMR}\left(\mathrm{CDCl}_{3}\right) \delta: 18.94 .{ }^{1} \mathrm{H}-\mathrm{NMR}\left(\mathrm{CDCl}_{3}\right) \delta: 7.13-7.78(\mathrm{~m}, 10 \mathrm{H}, \mathrm{Ar}-$ $\mathrm{H}),\left[5.37-5.47(\mathrm{~m}, \mathrm{Ha}), 4.96-5.03(\mathrm{~m}, \mathrm{Hb}) ; 4-\mathrm{CH}_{2}\right], 4.23-4.28(\mathrm{~m}, 2 \mathrm{H}$, $\left.\mathrm{CH}_{2} \mathrm{Cl}\right), 2.67-2.75\left(\mathrm{~m}, 2 \mathrm{H}, \mathrm{P}-\mathrm{CH}_{2}\right) .{ }^{13} \mathrm{C}-\mathrm{NMR}\left(\mathrm{CDCl}_{3}\right) \delta: 51.4(\mathrm{C}-4)$, 130.7 (C-5), 121.9 (C-6), 129.6 (C-7), 129.0 (C-8), 127.5 (C-9), 119.0 (C10), 149.6 (C-11), 114.5 (C-12), 132.8 (C-13), 128.5 (C-14), 147.1 (C-1'), $118.7\left(\mathrm{C}-2^{\prime}, 6^{\prime}\right), 132.5\left(\mathrm{C}-3^{\prime}, 5^{\prime}\right), 112.2\left(\mathrm{C}-4^{\prime}\right), 32.4\left(\mathrm{~d},{ }^{1} J_{\mathrm{PC}}=131.9 \mathrm{~Hz}, \mathrm{C}-\right.$ 1", P- $\left.\mathrm{CH}_{2}\right), 36.7\left(\mathrm{C}-2^{\prime \prime}, \mathrm{CH}_{2} \mathrm{Cl}\right)$. GC-MS m/z (\%): 437 [27, $\left.\left(\mathrm{M}^{+}+2\right)\right], 435$ $\left(19,\left(\mathrm{M}^{+}\right)\right], 372$ (28.5), 326 (13.3), 325 (11.4), 309 (65), 293 (9), 230 (65), 184 (19), 173 (21), 171 (19), 156 (18), 144 (28), 128 (60), 83 (100).

2-(2"-Chloroethyl)-2,3-dihydro-5-benzoyl-1 H-1,3,2-benzodiazaphosphole 2-Oxide (14) Yield: $0.33 \mathrm{~g}(52 \%)$; $\mathrm{mp} 158-160^{\circ} \mathrm{C}(\mathrm{d})$. Anal. Calcd for $\mathrm{C}_{15} \mathrm{H}_{14} \mathrm{~N}_{2} \mathrm{PO}_{2} \mathrm{Cl}$ : C, 56.17; H, 4.39; N, 8.73. Found : $\mathrm{C}, 56.28 ; \mathrm{H}, 4.52$; $\mathrm{N}$, 8.61. IR $(\mathrm{KBr}) \mathrm{cm}^{-1}: 1260(\mathrm{P}=\mathrm{O}), 797(\mathrm{P}-\mathrm{C}), 3352(\mathrm{P}-\mathrm{NH}), 1621$ $(\mathrm{C}=\mathrm{O}) .{ }^{31} \mathrm{P}-\mathrm{NMR}\left(\mathrm{DMSO}-d_{6}\right) \delta: 21.22 .{ }^{1} \mathrm{H}-\mathrm{NMR}\left(\mathrm{DMSO}-d_{6}\right) \delta: 7.07(\mathrm{~s}, 4-$ $\mathrm{H}), 6.55(\mathrm{~d}, J=7.8 \mathrm{~Hz}, 6-\mathrm{H}), 6.90(\mathrm{~d}, J=8.1 \mathrm{~Hz}, 7-\mathrm{H}), 7.48-7.59(\mathrm{~m}, 5 \mathrm{H}$, benzoyl-H), $3.67-3.72\left(\mathrm{~m}, 2 \mathrm{H}, \mathrm{CH}_{2} \mathrm{Cl}\right), 2.68-2.76\left(\mathrm{~m}, 2 \mathrm{H}, \mathrm{P}-\mathrm{CH}_{2}\right)$.

2-(Allyl)-2,3-dihydro-5-benzoyl-1 $H-1,3,2$-benzodiazaphosphole 2Oxide (15) Yield: $0.36 \mathrm{~g}(60 \%) ; \mathrm{mp} 149-151^{\circ} \mathrm{C}$. Anal. Calcd for $\mathrm{C}_{16} \mathrm{H}_{15} \mathrm{~N}_{2} \mathrm{PO}_{2}$ : C, 64.42; H, 5.06; N, 9.39. Found: $\mathrm{C}, 64.54 ; \mathrm{H}, 5.26 ; \mathrm{N}, 9.27$. IR $(\mathrm{KBr}) \mathrm{cm}^{-1}: 1262(\mathrm{P}=\mathrm{O}), 1604(\mathrm{C}=\mathrm{O}), 3356(\mathrm{P}-\mathrm{NH}), 728(\mathrm{P}-\mathrm{C}) .{ }^{31} \mathrm{P}-$ NMR (DMSO- $\left.d_{6}\right) \delta: 24.37 .{ }^{1} \mathrm{H}-\mathrm{NMR}$ (DMSO- $\left.d_{6}\right) \delta: 6.93(\mathrm{~s}, 4-\mathrm{H}), 6.72(\mathrm{~d}$, $J=7.9 \mathrm{~Hz}, 6-\mathrm{H}), 7.00(\mathrm{~d}, J=7.2 \mathrm{~Hz}, 7-\mathrm{H}), 7.18-7.59$ (m, 7H, benzoyl-H+ olefinic-H), $9.08(\mathrm{brs}, 2 \mathrm{H}, \mathrm{NH}), 3.66-3.87\left(\mathrm{~m}, 2 \mathrm{H}, \mathrm{P}-\mathrm{CH}_{2}\right), 5.29-5.89$ $\left(\mathrm{m},{ }^{1} \mathrm{H}, \mathrm{CH}\right), 5.26\left(\mathrm{~d}, J_{\text {trans }}=18 \mathrm{~Hz}, \mathrm{CH}_{2}\right), 5.64\left(\mathrm{~d}, J_{c i s}=11.6 \mathrm{~Hz}, \mathrm{CH}_{2}\right)$. EI-MS $\mathrm{m} / z$ (\%): $298\left(35, \mathrm{M}^{+}\right), 241$ (20), 212 (7), $136(100), 107$ (50), 105 (30).

4-(Phenyl)-2-(2"-chloroethyl)-1H-1,3,3a,5,6-pentaza-2-phosphapentalene 2-Oxide (17) Yield: $0.25 \mathrm{~g}(44 \%) ; \mathrm{mp} 238-240^{\circ} \mathrm{C}$. Anal. Calcd for $\mathrm{C}_{10} \mathrm{H}_{11} \mathrm{~N}_{5} \mathrm{POCl}$ : C, 42.34; H, 3.90; N, 24.68. Found: $\mathrm{C}, 42.18 ; \mathrm{H}, 3.69 ; \mathrm{N}$ 24.45. IR (KBr) cm ${ }^{-1}: 1205(\mathrm{P}=\mathrm{O}), 782(\mathrm{P}-\mathrm{C}), 3363$ (P-NH). ${ }^{31} \mathrm{P}-\mathrm{NMR}$ $\left(\mathrm{DMSO}-d_{6}\right) \delta: 24.17 .{ }^{1} \mathrm{H}-\mathrm{NMR}\left(\mathrm{DMSO}-d_{6}\right) \delta: 7.17-7.79(\mathrm{~m}, 5 \mathrm{H}, \mathrm{Ar}-\mathrm{H})$, $13.83(\mathrm{~d}, J=24.7 \mathrm{~Hz}, \mathrm{~N}-\mathrm{NH}), 9.11(\mathrm{~s}, \mathrm{P}-\mathrm{NH}), 2.02-2.30\left(\mathrm{~m}, 2 \mathrm{H}, \mathrm{P}-\mathrm{CH}_{2}\right)$, $3.09-3.73\left(\mathrm{~m}, 2 \mathrm{H}, \mathrm{CH}_{2} \mathrm{Cl}\right) .{ }^{13} \mathrm{C}-\mathrm{NMR}\left(\mathrm{DMSO}-d_{6}\right) \delta: 157.4(\mathrm{C}-4, \mathrm{C}=\mathrm{N})$, $157.7(\mathrm{C}-6 \mathrm{a}, \mathrm{C}=\mathrm{N}), 144.9\left(\mathrm{C}-1^{\prime}\right), 125.6\left(\mathrm{C}-2^{\prime}, 6\right), 129.0\left(\mathrm{C}-3^{\prime}, 5^{\prime}\right), 129.8$ $\left(\mathrm{C}-4^{\prime}\right), 29.6\left(\mathrm{~d},{ }^{1} J_{\mathrm{PC}}=133.4 \mathrm{~Hz}, \mathrm{C}-1^{\prime \prime}, \mathrm{P}-\mathrm{CH}_{2}\right), 45.8\left(\mathrm{C}-2^{\prime \prime}, \mathrm{CH}_{2} \mathrm{Cl}\right)$.

4-(Benzyl)-2-(2"-chloroethyl)-1 H-1,3,3a,5,6-pentaza-2-phospha-pentalene 2-Oxide (19) Yield: $0.22 \mathrm{~g}(42 \%)$; mp 206-208 ${ }^{\circ} \mathrm{C}$. Anal. Calcd for $\mathrm{C}_{11} \mathrm{H}_{13} \mathrm{~N}_{5} \mathrm{POCl}: \mathrm{C}, 44.38 ; \mathrm{H}, 4.40 ; \mathrm{N}, 23.52$. Found: $\mathrm{C}, 44.52 ; \mathrm{H}, 4.27 ; \mathrm{N}$, 23.63. IR ( KBr) cm ${ }^{-1}: 1258(\mathrm{P}=\mathrm{O}), 3210(\mathrm{P}-\mathrm{NH}), 803(\mathrm{P}-\mathrm{C}) .{ }^{31} \mathrm{P}-\mathrm{NMR}$ $\left(\right.$ DMSO- $\left.d_{6}\right) \delta: 22.33 .{ }^{1} \mathrm{H}-\mathrm{NMR}$ (DMSO- $\left.d_{6}\right) \delta: 7.01-7.36(\mathrm{~m}, 4 \mathrm{H}, \mathrm{Ar}-\mathrm{H})$, $4.63\left(\mathrm{~s}, 2 \mathrm{H}, 1^{\prime}-\mathrm{CH}_{2}\right), 10.19$ (d, $\left.J=24.3 \mathrm{~Hz}, \mathrm{~N}-\mathrm{NH}\right), 6.30$ (s, 1H, P-NH), $2.14-2.29\left(\mathrm{~m}, 2 \mathrm{H}, \mathrm{P}-\mathrm{CH}_{2}\right), 3.68-4.06\left(\mathrm{~m}, 2 \mathrm{H}, \mathrm{CH}_{2} \mathrm{Cl}\right)$.

Acknowledgements The authors express their thanks to Dr. C. Naga Raju, Department of Chemical Engineering, Sri Venkateswara University, Tirupati for his fruitful discussions. Ms. M. D. Kamar Jahan, Research Scholar, Department of Botany, Sri Venkateswara University, Tirupati, for her help in antimicrobial studies. Assistance of Dr. M. R. Ashok Associate Professor of English has been sought for proof correction of the paper. One of the authors P. V. G. Reddy wishes to thank CSIR, New Delhi, India for the award of Senior Research Fellowship. The authors are also thankful to the UGC, New Delhi, for providing the Financial Assistance.

\section{References}

1) Johnson A. W., Kasa W. C., Ostoza K. A., Dixon D. A., "Ylides and Imines of Phosphorus," Wiley, New York, 1993.

2) Miller P., Mol. Cell. Biol., 1, 83-88 (1992).

3) Blackburn G. M., Chem. Ind., 5, 134-137 (1981).

4) Hogland R. E., ACS Sym. Ser., 380, 182-188 (1988).

5) Coffey M. D., Ouimette D. G., Symo. Br. Mycol. Soc., 1988, 107-110 (1988).

6) Kasting G. B., Francis M. D., J. Bone Miner. Res., 7, 513-516 (1992).

7) Denmark S. E., Dorow R. L., J. Org. Chem., 55, 5926-5931 (1990).

8) Hua D. H., Chen I. S., Saha S., Wang H., Roche D., Bharathi S. N., Chan-Yu-King R., Robinson R. D., Iguchi S., Syn. Lett., 1992, 817820 (1992).

9) Reddy B. S., Reddy C. D., Reddy P. M., Heteroatom Chem., 5, 507511 (1994).

10) Babu Y. H., Reddy P. V. G., Reddy C. S., Reddy C. D., Devi P. U. M., $J$ Heterocyclic Chem., 39, 1039-1044 (2002).

11) Srinvasulu D., Reddy C. D., Reddy C. S., Brown C. W., Hager J. D., Means M., Berlin K. D., Phosphorus, Sulfur and Silicon, 167, 181193 (2000).

12) Reddy P. V. G., Babu Y. H., Reddy C. S., Srinvasulu D., Hetero Atom Chem., 13, 340-345 (2002).

13) Kinnear A. M., Perren A. E., J. Chem. Soc., 1952, 3437-3444 (1952).

14) Burke W. J., J. Am. Chem. Soc., 71, 609-612 (1949).

15) Burke W. J., Murdock K. C., Grace E. C., J. Am. Chem. Soc., 76, 1677-1679 (1954)

16) Padhy A. K., Nag V. L., Panda C. S., Indian J. Chem., 38B, 998-1001 (1999).

17) Kochhar M. M., J. Heterocyclic Chem., 1971, 153-154 (1971).

18) Reddy C. D., Berlin K. D., Reddy R. S., Raju C. N., Elmasri M., Subramanian S., Phosphorus, Sulfur and Silicon, 81, 61-67 (1993).

19) Reddy C. D., Reddy R. S., Raju C. N., Elmasri M., Berlin K. D., Surbamanian S., Magn. Reson. Chem., 29, 1140-1145 (1991).

20) Pastor S. D., Spivack J. D., J. Heterocyclic Chem., 28, 1561-1565 (1991).

21) Goddard J. D., Payne A. W., Cook M., J. Heterocyclic Chem., 25 $575-578$ (1988).

22) Cameron T. S., Cardos F. E., Demir T., Shaw R. A., J. Chem. Soc., Perkin Trans. 1, 1979, 2986-2989 (1979).

23) Colle J. G., Duguid J. P., Fraser A. G., Marmion B. P., "Mackie \& Mc Cartney Practical Medical Microbiology," 13th ed., Vol. 2, Churchil Livingstone, London, 1989.

24) Devi P. U. M., Reddy P. S., Usharani N. R., Reddemma P., Eur. J. Plant Pathol., 106, 857-865 (2000).

25) Silverstein R. M., Bassler G. C., Morril T. C., "Spectrometric Identification of Organic Compounds," John Wiley \& Sons, New York, 1991. 